Disclosure of Interest: None declared

DOI: 10.1136/annrheumdis-2018-eular.4093

\section{SAT0521 A PROSPECTIVE OBSERVATIONAL STUDY ON THE SAFETY AND EFFICACY OF INFLIXIMAB-BIOSIMILAR IN PATIENTS WITH TAKAYASU'S ARTERITIS (TAKASIM): PRELIMINARY DATA}

C. Campochiaro, A. Tomelleri, S. Sartorelli, C. Sembenini, G. Cavalli, E. Baldissera, L. Dagna. Unit of Rheumatology, Immunology, Allergy and Rare Diseases (UnIRAR), San Raffaele Scientific Institute, Milan, Italy

Background: Takayasu arteritis(TA) is a large-vessel vasculitis ${ }^{1}$. Treatment is mainly based on steroids, but in approximately $50 \%$ of patients a disease-modifying antirheumatic drug(DMARD) is required ${ }^{2}$. Anti-TNF $\alpha$ agents are recommended for steroid tapering despite DMARDs ${ }^{3}$. Infliximab-originator(IFX-O) is a chimeric monoclonal antibody against TNF $\alpha$ effective in TA patients. Infliximabbiosimilar(IFX-B) is an immunoglobulin-G1 chimeric human-murine monoclonal antibody biosimilar to IFX-O.

Objectives: To assess safety and efficacy of IFN-B in TA patients requiring antiTNF $\alpha$ therapy.

Methods: 30 TA patients, diagnosed according to ACR criteria at our tertiary centre, will be recruited from our cohort. Both biological therapy-naïve and IFX-O treated patients will be eligible. Disease activity will be assessed 6-monthly by means of magnetic resonance angiography(MRA) and $18 \mathrm{~F}$-fluorodeoxyglucose (FDG) PET/CT. ITAS2010 and ITAS-ESR/CRP will be obtained 6-monthly. Baseline was defined as the time of IFX-B start. Non parametric statistic tests were used.

Results: At January 2018, 19 patients(18 female, 1 male) were included. 12 patients have been on IFX-B for at least 6 months. Median age at baseline was 45 years(range 25-70). At recruitment median disease duration was 52 months (range 24-180), all patients were on IFX-O. Median time on IFX-O at baseline was 35 months(range14-150). 3 patients had been previously treated with other biologics: tocilizumab $\left({ }^{2}\right.$ adalimumab $\left({ }^{1}{ }^{1} 18 / 19\right.$ patients $(94.7 \%)$ were on concomitant steroid therapy (mean dose $5 \pm 1.8 \mathrm{mg}$ ). It was significantly reduced to a mean dose of $4 \pm 1.7 \mathrm{mg}(\mathrm{p}=0.043)$ at month $6.15 / 19$ patients $(78.9 \%)$ were also on DMARDs, kept unchanged throughout treatment. 1 patient on IFX-B was switched to a different therapy because of poor disease control with both IFX-O and IFX-B. Mean IFX-B dose at baseline was $6.92 \pm 1.76 \mathrm{mg} / \mathrm{kg}$. Mean IFX-B dose at month 6 was $7.42 \pm 2.19 \mathrm{mg} / \mathrm{kg}$. IFX-B dose was increased in 5 patients. Mean time interval between IFX-B infusions was kept unchanged(5.79 \pm 0.63 weeks). Mean CRP and ESR were $3.28 \pm 2.64 \mathrm{mg} / \mathrm{L}$ and $19.68 \pm 9.94 \mathrm{~mm} / 1$ hour at baseline and 3.4 $\pm 3.12 \mathrm{mg} / \mathrm{L}$ and $20.53 \pm 14.06 \mathrm{~mm} / 1$ hour at month 6 , the difference not being statistically significant. Mean ITAS2010 and ITAS-ESR/CRP were 7.6, 8.2 and 8 at baseline and $6.1,6.8$ and 6.3 at month 6 , the difference not being statistically significant. At month $6 \mathrm{PET} / \mathrm{CT}$ showed no disease progression in all patients and MRA disclosed disease stability in $8 / 12(66.7 \%)$, progression in $3 / 12(25 \%)$ and improvement in $1(8.3 \%)$ patient. No patient experienced side effects during infusion. $11 / 19$ patients $(57.9 \%)$ experienced low-grade side effects related to TNF $\alpha$ blockade. 6 patients experienced upper airway infection, 3 herpes simplex reactivation, 1 viral gastroenteritis, 1 vaginal candidiasis. No modification of IFX-B therapy was required.

Conclusions: Our preliminary data suggest that IFX-B is as effective and safe as IFX-O in TA patients.

\section{REFERENCES:}

[1] Numano F, et al. Takayasu's arteritis. Lancet 2000.

[2] Hoffman GS, et al. Treatment of glucocorticoid-resistant or relapsing Takayasu arteritis with methotrexate. Arthritis Rheum 1994.

[3] Clifford A, et al. Recent advances in the medical management of Takayasu arteritis: an update on use of biologic therapies. Current Opinion in Rheumatology 2014.

Disclosure of Interest: C. Campochiaro: None declared, A. Tomelleri: None declared, S. Sartorelli: None declared, C. Sembenini: None declared, G. Cavalli: None declared, E. Baldissera: None declared, L. Dagna Grant/research support from: The Unit has received unrestricted educational grants from Abbvie, BMS, Celgene, Mundipharma, Novartis, MSD, Pfizer, Roche, and SOBI.

DOI: 10.1136/annrheumdis-2018-eular.5543

\section{SAT0522}

VASCULAR AND JOINT INFLAMMATION ARE NEGATIVELY CORRELATED IN PATIENTS WITH POLYMYALGIA RHEUMATICA, GIANT CELL ARTERITIS AND FEVER OF UNKNOWN ORIGIN

D. Camellino ${ }^{1,2}$, M. Bauckneht ${ }^{3}$, S. Morbelli ${ }^{4}$, M. Marchiano ${ }^{2}$, G. Pesce ${ }^{2}$, D. Saverino ${ }^{2}$, M. Bagnasco ${ }^{2}$, M.A. Cimmino ${ }^{5} .{ }^{1}$ Division of Rheumatology, Musculoskeletal System Department, Azienda Sanitaria Locale 3 Genovese, Arenzano; ${ }^{2}$ Autoimmunology Laboratory, Department of Internal Medicine; ${ }^{3}$ Nuclear Medicine Division, Department of Health Sciences; ${ }^{4}$ University of Genoa, Genoa, Italy; ${ }^{5}$ Rheumatology Clinic, Department of Internal Medicine, University of Genoa, Genoa, Italy

Background: 18 F-Fluorodeoxyglucose positron emission tomography (PET) reveals the presence of large vessel vasculitis (LVV) in $30 \%-40 \%$ of patients with apparently isolated polymyalgia rheumatica (PMR), in $70 \%-80 \%$ of patient with giant cell arteritis ${ }^{[1]}$ and in about $20 \%$ of patient with fever of unknown origin $(\mathrm{FUO})^{[2]}$, suggesting that these conditions may be different clinical manifestations of the same entity.

Objectives: To evaluate and compare the patterns of vascular and joint uptake in patients with PMR, GCA, and FUO.

Methods: Consecutive patients with a diagnosis of PMR, GCA or FUO underwent a thorough clinical examination and a PET/CT scan. Arterial and joint uptake of FDG were scored relative to liver uptake with a 4-point scale. The values of each district examined were summed to obtain a total vascular score (TVS) and a total joint score (TJS). A semi-quantitative analysis of FDG uptake was carried out. Arterial FDG uptake was quantified by calculating the mean standardised uptake value (SUV) within each region of interest $(\mathrm{ROI})$ and the results expressed as the ratio between mean SUV value of each $\mathrm{ROI}$ and the blood-bool (SUV/BP). To assess joint metabolism, CT-based ROls were bilaterally drawn on joint and bursal spaces.

Results: One hundred and thirty-one patients were included, 89 females and 42 males, with a median age of 74 years (range 47-92). Ninety-seven patients were diagnosed as PMR, 13 as GCA, 16 with both PMR and GCA and 5 patients presented with FUO. FUO patients showed a higher mean arterial SUV in comparison to PMR (0.77 vs. $1.15, p=0.004)$ and GCA patients $(0.81$ vs $1.15, p=0.052)$ Similar and more striking results were obtained using visual scoring. FUO patients showed always increased uptake in the large vessels. PMR patients showed statistically lower TVS than GCA +PMR, GCA and FUO patients. Patients with PMR showed a statistically significant higher mean joint SUV than GCA patients $(p=0.01)$. The highest mean articular uptake was shown by PMR patients and the lowest by FUO patients. Mean TJS of PMR patients was significantly higher than that of GCA and FUO patients $(p<0.001)$. TVS and TJS correlated negatively $(p=0.01)$, as did mean vascular SUV and mean joint SUV $(p=0.001)$.

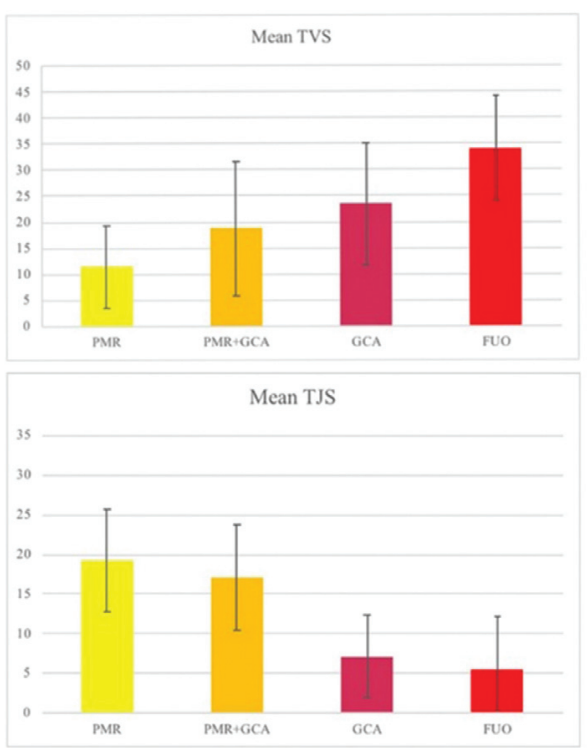

Conclusions: Although patients with diseases different from PMR were few, our PET/CT study support the view that there is a continuum in the intensity of inflammation, with FUO >GCA>PMR+GCA > PMR for vessels, and the opposite for joints. Vascular and joint inflammation were negatively correlated. Our data support the view that similarities exceed differences in these conditions, with clinical features depending from the relative contribution of vessel and joint involvement. 